\section{THU0411 TOPHUS BURDEN. CLINICAL EVALUATION DURING 3 YEARS FOLLOW-UP IN GRESGO COHORT}

A. Maya Gutierrez ${ }^{1}$, C.O. Lopez Lopez ${ }^{2}$, E. Alvarez Hernandez ${ }^{1}$, C. Gomez Ruiz $^{1}$, I. Peláez Ballestas ${ }^{1}$, R. Burgos-Vargas ${ }^{1}$, J. Vazquez Mellado ${ }^{1,3}$.

${ }^{1}$ Rheumatology; ${ }^{2}$ Medicina Física y Rehabilitación, Hospital General de México; ${ }^{3}$ Facultad de Medicina, UNAM, Mexico, Mexico

Background: Tophus burden (TB) was proposed by OMERACT as mandatory domain for chronic gout studies. Image methods although useful, frequently are not available in daily clinical practice.

Objectives: To evaluate TB clinically in a cohort of gout patients during 3 years follow-up.

Methods: We analyzed baseline and follow-up data $(6,12,18,20,24,30$ and 36 months) of patients with gout (ARA/CGD/ACR-EULAR) from the GRESGO cohort; this analysis includes the patients with $>1$ clinical nodule(s) considered as tophi in the baseline visit. Index tophi size (ITS) was considered as the longer axis of the bigger tophi. TB evaluations: 1) Tophi number (T); 2) ITSt (cm) measured with tape; 3) ITSca (cm), measured with caliper; 4) ITSco, measured with compass. 5) Index tophi hardness (ITh) or consistency, by VAS (0: very soft-10 hard as a rock). This protocol was approved by the local IRB and patients signed and informed consent for their participation.

All the patients received treatment for gout and associated diseases according to published guides and available drugs. The evaluations were done by the same group of physicians (Rheumatologists and Residents). Variables: TB measures, demographic, clinical and biochemical variables. HAQ and EUROQ pain and general health. Inter/intraobserver variability were evaluated. Statistical analysis: $t$ test, $\mathrm{X} 2$, r, kappa, Friedman and multiple correlation.

Results: 298 patients; 97\%males, $203(68 \%)$ tophaceous gout, age, age at onset and duration of the disease were: $46.4 \pm 12.7 \quad 32.4 \pm 12.4$ and $14.7 \pm 9.3$ years respectively. Available ULT: Allopurinol and probenecid, colchicine as prophylaxis. Mean allopurinol dose prescribed at baseline visit was $346.9 \pm 154$ $\mathrm{mg} / \mathrm{d}$ and $550 \pm 205 \mathrm{mg} / \mathrm{d} 3$ years later.TB values (see table). Index tophi size had $r>0.93$ among the 3 evaluations (ITSt, ITSca, ITSco) and $r<0.3$ with ITh. Inter and intraobserver variability was 0.8 and 0.9 respectively; there was significant improvement ${ }^{*}(p<0.001)$ in ITS, sUA, painful, swollen, limited to motion joints, $\mathrm{HAQ}$, EuROQoL, VAS pain, VAS health (patient and physician) also improved significantly.

\begin{tabular}{lccccccc}
\hline & Baseline & $6 \mathrm{mo}$ & $12 \mathrm{mo}$ & $18 \mathrm{mo}$ & $24 \mathrm{mo}$ & $30 \mathrm{mo}$ & $36 \mathrm{mo}$ \\
\hline ITSt $^{*}$ & $6.4 \pm 3.7$ & $5.9 \pm 3.7$ & $5.6 \pm 3.7$ & $5.7 \pm 3.9$ & $5.2 \pm 4.0$ & $5.6 \pm 3.3$ & $4.5 \pm 3.3$ \\
ITSca & $5.1 \pm 2.8$ & $4.6 \pm 2.7$ & $4.7 \pm 2.6$ & $4.7 \pm 2.9$ & $4.4 \pm 2.7$ & $4.6 \pm 2.4$ & $3.6 \pm 1.9$ \\
ITSco & $5.1 \pm 3.0$ & $4.6 \pm 2.8$ & $4.7 \pm 2.7$ & $4.9 \pm 3.0$ & $4.3 \pm 2.8$ & $4.6 \pm 2.5$ & $3.8 \pm 1.8$ \\
ITh & $7.3 \pm 1.7$ & $6.8 \pm 1.9$ & $7.1 \pm 1.6$ & $6.7 \pm 1.9$ & $6.5 \pm 1.8$ & $6.6 \pm 2.0$ & $6.3 \pm 1.6$ \\
$\mathrm{HAQ}^{*}$ & $0.6 \pm 0.7$ & $0.4 \pm 0.5$ & $0.3 \pm 0.5$ & $0.4 \pm 0.4$ & $0.3 \pm 0.4$ & $0.4 \pm 0.5$ & $0.2 \pm 0.4$ \\
AGA $^{*}$ & $8.3 \pm 12$ & $0.8 \pm 1.3$ & $0.6 \pm 2.6$ & $0.3 \pm 0.8$ & $0.2 \pm 0.6$ & $0.2 \pm 0.4$ & $0.3 \pm 0.6$ \\
sUA* $_{\text {Allopurinol* }}^{*}$ & $8.3 \pm 2.2$ & $7.3 \pm 2.0$ & $6.8 \pm 1.9$ & $6.3 \pm 1.9$ & $6.3 \pm 2.5$ & $5.5 \pm 1.6$ & $5.8 \pm 1.8$ \\
& $347 \pm 154$ & $445 \pm 165$ & $477 \pm 178$ & $491 \pm 180$ & $534 \pm 205$ & $504 \pm 183$ & $556 \pm 193$ \\
\hline
\end{tabular}

Values represent mean and SD. ${ }^{*} \mathrm{p}<0.01$ ITS in $\mathrm{cm}$; ITh: VAS in $\mathrm{cm}$. AGA number of acute attacks in the last 6 months. sUA: $\mathrm{mg} / \mathrm{dL}$, allopurinol dose $\mathrm{mg} /$ day.

Conclusions: In our cohort, ITSt was the best measure for TB evaluation, the 3 ITS measures had a good correlation between them and less with ITh or consistency. Patients with tophaceous gout, improved significantly with allopurinol and probenecid during 3 years follow-up in TB (ITSt) as well according to the other OMERACT proposed domains for chronic gout.

Disclosure of Interest: None declared

DOI: 10.1136/annrheumdis-2017-eular.2142

\section{THU0412 TREATMENT WITH NERIDRONATE IN CHILDREN AND ADULTS WITH OSTEOGENESIS IMPERFECTA: DATA FROM OPEN-LABEL, NOT CONTROLLED, THREE-YEAR ITALIAN STUDY}

A. Fassio, L. Idolazzi, O. Viapiana, C. Benini, E. Vantaggiato, A. Giollo, M. Rossini, D. Gatti. Rheumatology, Aoui Verona Reumatologia, Verona, Italy

Background: Osteogenesis Imperfecta $(\mathrm{OI})$ is a rare generalized connective tissue disease. Its main features are skeletal fragility and substantial growth deficiency [1]. Currently, bisphosphonates showed to increase bone mineral density (BMD). A positive effect on prevention of fractures both in adults and in children is reported by some studies, but generally data are still inconsistent [2]. Neridronate is an amino-bisphosphonate licensed in Italy for the treatment of OI. Objectives: to assess the long-term efficacy and safety of the treatment in patients with OI.

Methods: the patients were divided by age into two groups and observed for 3 years: 55 patients younger than 20 years old and 114 patients older than 20 years old. Neridronate was administered by i.v. infusion at the dosage of $2 \mathrm{mg} / \mathrm{kg}$, up to a maximum of $100 \mathrm{mg}$ at three months intervals. DXA of the lumbar spine, hip and ultradistal radius were evaluated every 6 months. Blood calcium, phosphate, bone turnover markers and fasting urinary calcium/creatinine ratio, were obtained at baseline and every 3 months.

Results: the mean lumbar spine and total hip BMD and BMC significantly increased from baseline up to month 36 in both patients groups. The mean ultradistal radius BMD significantly increased from baseline to any time point in patients younger than 20 years, while, in patients older than 20 years, BMD significantly increased from baseline only at month 18,30 and 36 respectively. The mean ultradistal radius BMC significantly increased from baseline to any time point in patients younger than 20 years, while there were no substantial or statistically significant changes from baseline to any time point in patients aged older than 20 years. The mean number of fractures observed in the 3 years of treatment was significantly lower than that observed in the 3 years before the start of treatment in both groups (table 1).

Most of AEs were symptoms of an acute phase reaction, which was reported in $47.3 \%$ of patients younger than 20 years and in $22.8 \%$ of those older than 20 years. Serious adverse events (SAEs) were reported in 19 patients (34.5\%) younger than 20 years and in 26 patients $(22.8 \%)$ aged older than 20 years. None of the reported SAEs in both groups was considered as treatment-related.

Table 1, Results of number of fractures per patient during treatment in the two patient populations

\begin{tabular}{lcc}
\hline Number of fractures, mean \pm SD (range) & Age $\leq 20$ years & Age $>20$ years \\
\hline Before treatment ( 3 years & $2.7 \pm 2.37(0-8)$ & $0.6 \pm 1.28(0-8)$ \\
During treatment ( 3 years) & $0.9 \pm 1.43(0-7)$ & $0.3 \pm 0.56(0-3)$ \\
- Wilcoxon signed rank test p-value & $<0.001$ & 0.003 \\
\hline
\end{tabular}

Conclusions: long-term treatment with i.v.neridronate has positive effects on $\mathrm{BMD}, \mathrm{BMC}$, bone turnover markers and fracture risk with a good safety profile in both groups.

References:

[1] Hoyer-Kuhn H, Netzer C, Semler O. Osteogenesis imperfecta: pathophysiology and treatment. Wien Med Wochenschr 1946 2015;165:278-84 doi:10.1007/s10354-015-0361-x.

[2] Hald JD, Evangelou E, Langdahl BL, et al. Bisphosphonates for the Prevention of Fractures in Osteogenesis Imperfecta: Meta-Analysis of Placebo-Controlled Trials. J Bone Miner Res 2015;30:929-33. doi:10.1002/jbmr.2410.

Disclosure of Interest: None declared

DOI: 10.1136/annrheumdis-2017-eular.2717

\section{THU0413 SERUM URATE AND ITS ASSOCIATION WITH BLOOD PRESSURE AND ENDOTHELIAL DYSFUNCTION IN YOUNG ADULTS}

M.B. Saddekni ${ }^{1}$, K.G. Saag ${ }^{1}$, T. Dudenbostel ${ }^{1}$, D.A. Calhoun ${ }^{2}$, S. Oparil ${ }^{2}$, D.I. Feig ${ }^{3}$, P. Muntner ${ }^{4}$, P.J. Foster ${ }^{1}$, S.R. Biggers ${ }^{5}$, E.J. Rahn ${ }^{5}$, P. Li ${ }^{6}$ D.T. Redden ${ }^{6}$, A.L. Gaffo ${ }^{5}$. ${ }^{1}$ UAB School of Medicine, Division of Clinical Immunology and Rheumatology; ${ }^{2}$ UAB School of Medicine, Division of Cardiovascular Disease; ${ }^{3}$ UAB School of Medicine Division of Nephrology; ${ }^{4} U A B$ School of Public Health, Department of Epidemiology; ${ }^{5}$ Department of Medicine, Division of Rheumatology and Immunology; ${ }^{6}$ UAB School of Public Health, Department of Biostatistics, University of Alabama at Birmingham, Bimringham, United States

Background: Both serum urate (sUA) and endothelial dysfunction have been associated with hypertension and cardiovascular disease. Increasing SUA level has been associated with endothelial dysfunction and higher inflammatory markers. To date, however, few studies have examined the relationship between sUA, endothelial dysfunction, and blood pressure (BP) including young and largely healthy individuals.

Objectives: To determine whether there is an association between higher sUA, endothelial dysfunction as measured by flow-mediated dilation (FMD), and BP in young adults.

Methods: We conducted a cross-sectional analysis of baseline data for consecutively enrolled individuals (age $18-40$ years). Enrollment criteria included baseline systolic BP (SBP) $\geq 120$ and $<160 \mathrm{mmHg}$ or diastolic $\mathrm{BP}$ (DBP) $\geq 80$ and $<100 \mathrm{mmHg}$, and $\mathrm{s} U A \geq 5.0 \mathrm{mg} / \mathrm{dL}$ for men or $\geq 4.0 \mathrm{mg} / \mathrm{dL}$ for women. Endothelial dysfunction measured by FMD, 24- hours ambulatory BP monitoring (ABPM) and sUA level were obtained. Associations between SUA, FMD, and ABPM variables were evaluated using a general linear model. Adjustments for age, gender, race, and BMI were applied after significant univariate results.

Results: 86 participants included in the analysis. Participants recruited had a mean age ( \pm standard deviation) of $28.5 \pm 6.9$ years, $36 \%$ were female, $41 \%$ African-Americans, mean BMI was $29.2+6.8 \mathrm{~kg} / \mathrm{m}^{2}$, and mean sUA was $5.9 \pm 1.2$ $\mathrm{mg} / \mathrm{dL}$ ( $\mathrm{n}=77$, range from 3.9 to $8.5 \mathrm{mg} / \mathrm{dL}$ ). We found no significant cross-sectional associations between SUA, FMD, and BP variables assessed by ABPM (Table). Participants in the upper tertile of sUA had significantly worse FMD than those in the lower tertiles (Figure). However, this difference was no longer significant after multivariable adjustment age, gender, race, and BMI.

Table 1. Cross-sectional correlation between SUA, FMD, and Ambulatory Blood Pressure parameters

\begin{tabular}{lcc}
\hline Parameters & $r$ & $p$-value \\
\hline sUA and FMD & -0.112 & 0.351 \\
SUA and SBP & 0.037 & 0.766 \\
SUA and DBP & -0.012 & 0.924 \\
sUA and MAP & 0.056 & 0.657 \\
\hline
\end{tabular}

Conclusions: In this cross-sectional analysis of young adults, there was no evidence to support an association between sUA levels and endothelial 


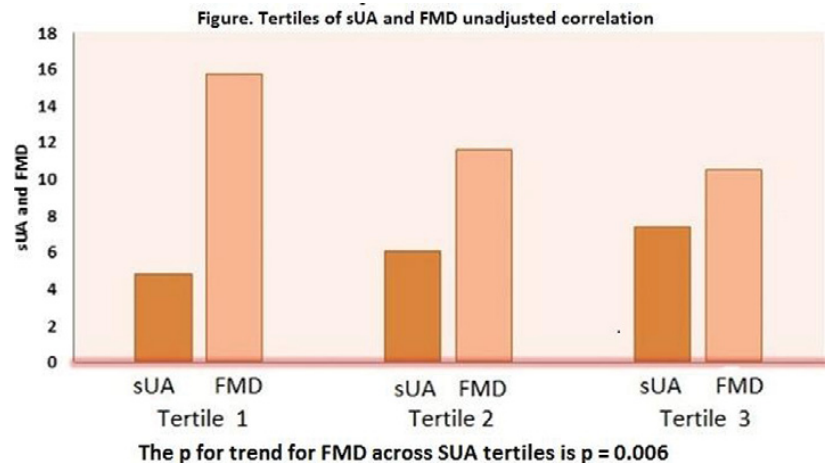

dysfunction or BP. Endothelial dysfunction or BP might be associated with changes in SUA when measured longitudinally in individuals, but not when measured cross-sectionally in populations. Larger studies will be needed to confirm these results.

References:

[1] Ruggiero, C. Am J Cardiol, 2007. 100(1): p. 115-21.

[2] Rossi, R. J Am Coll Cardiol, 2004. 44(8): p. 1636-40.

[3] Feig, D. JAMA, 2008. 300(8): p. 924-32.

Acknowledgements: National Institute of Arthritis and Musculoskeletal and Skin Diseases P50AR060772, K24AR052361 (to KGS).

Disclosure of Interest: M. Saddekni: None declared, K. Saag Grant/research support from: AstraZeneca, Crealta, Takeda, Consultant for: Ardea/AstraZeneca,

Crealta, Takeda, T. Dudenbostel: None declared, D. Calhoun: None declared, S. Oparil Grant/research support from: NHLBI Brigham and Women's Hospital, Center CVD Prev, Novartis Pharmaceutical Corporation, AstraZeneca AB (Duke University), Actelion Pharmaceuticals US,NIH/NHLBI, Merck and Co., Consultant for: Amgen, Bayer, Boehringer Ingelheim, AstraZeneca, Medtronic, GlaxoSmithKline, Forest Labs Inc., D. Feig: None declared, P. Muntner: None declared, P. Foster: None declared, S. Biggers: None declared, E. Rahn: None declared, P. Li: None declared, D. Redden: None declared, A. Gaffo Grant/research support from: Amgen, AstraZeneca, Consultant for: Cymabay, Ardea, Employee of: US Government

DOI: 10.1136/annrheumdis-2017-eular.5313

\section{THU0414 INCIDENCE AND PREDICTORS FOR NEPHROLITHIASIS IN GOUT PATIENTS AND THE GENERAL POPULATION}

A.J. Landgren ${ }^{1}$, L. Jacobsson ${ }^{1}$, U. Lindström ${ }^{1}$, T.Z. Sandström ${ }^{1}$, E. Fjellstedt ${ }^{2}$, P. Drivelegka ${ }^{1}$, V. Sigurdardottir ${ }^{1}$, L. Björkman ${ }^{1}$, M. Dehlin ${ }^{1}$. ${ }^{1}$ Department of Rheumatology and Inflammation Research, Institution of Medicine, Sahlgrenska Academy, University of Gothenburg, Gothenburg; ${ }^{2}$ Department of Nephrology and Transplantation, SUS University Hospital, Malmö, Sweden

Background: A well-known complication of gout is an increased risk for nephrolithiasis (NL). The incidence rate of $\mathrm{NL}$ in the general population varies in different studies between 85 and 170/100 000 person-years, with a peak incidence in the ages 40-49 years. Several medications used in gout patients could affect the risk for NL, including allopurinol, losartan, thiazide- and loop-diuretics. Effect of these medications on risk of NL in gout patients, and the general population, has only scarcely been studied.

Objectives: In this cohortstudy we investigated: 1) overall incidence of NL in gout and general population (GP) controls 2) risk for first time NL in gout patients vs general population (GP) controls, and 3) predictors for first time NL in both groups separately.

Methods: Gout patients were identified from the regional health care database in western Sweden (VEGA), containing ICD10-codes for all regional Healthcare visits from 2000. Matched (birthyear, sex, county) GP controls were selected from the population register. National registers and VEGA were used to retrieve information on comorbidities, socioeconomic factors and current medications at start of follow-up. The study population had to be above 19 years of age, without NL prior to start of follow-up, and living in the Western Swedish Health Care Region (WSHCR). Follow-up began 2006-01-01, or at the first gout-diagnosis if this occurred later, and ended at death, emigration or 2012-12-31, whichever occurred first. Incidence rates (IR) per 1000 person-years and hazardratios (HR) were calculated. Possible predictors for NL were based on risk factors presented in the literature.

Results: 29,968 gout patients and 138,678 matched GP controls were included. In gout patients there were $678 \mathrm{NL}$-events (IR: 6.2 per 1000 pyrs at risk $(95 \% \mathrm{Cl}$ : 5.7-6.6)) and in GP controls 2125 (IR 3.9 per 1000 pyrs at risk (95\% Cl 3.7-4.0)). Risk for NL was increased in gout ( $\mathrm{HR}=1.49,95 \% \mathrm{Cl}$ : $1.35-1.64)$, and was higher in men compared to women $(P<0.0001)$ in all age groups for gout cases and controls. All comorbidities and medications were more frequent in cases compared to controls $(p<0.0001)$ at start of follow-up. Risk-factors for NL such as kidney disease $(K D)$, obesity, diabetes were $2-4$ times more common in gout patients compared to GP controls. Predictor point estimates for NL were similar in gout cases and GP controls (Figure 1), except for losartan which increased the

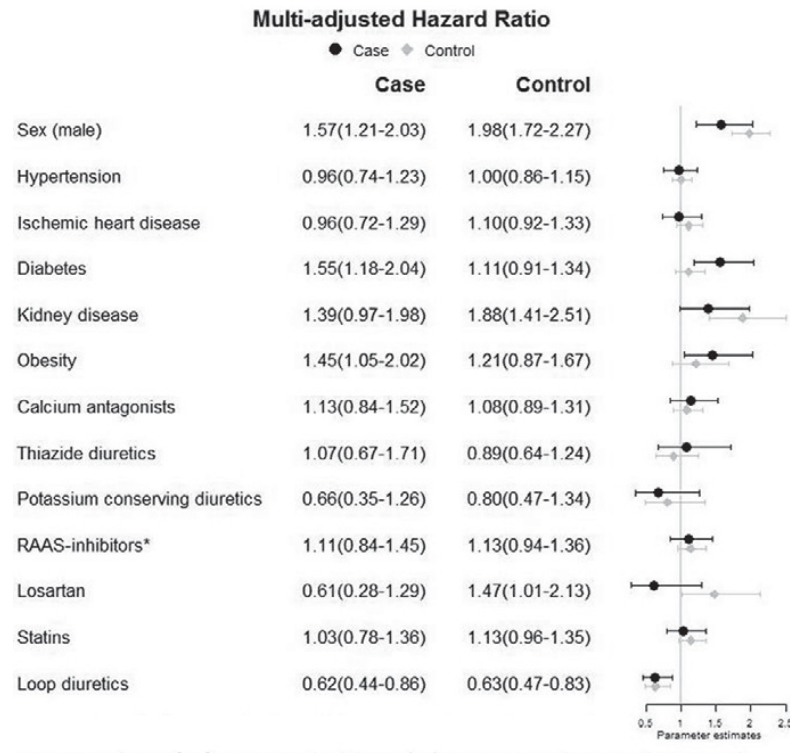

Figure 1 Predictors for first time nephrolithiasis (NL) in patients with gout and GP controls without gout, analyzed by multivariate proportional hazards analyses, adjusting for age and other covoriates in the figure, *Renin-angiotensin-aldosterone-System-Inhibitors excluding losortan

risk of NL in GP controls ( $\mathrm{HR}=1,47,95 \% \mathrm{Cl}: 1.01-2.13)$ but not in gout patients. Loop-diuretics appeared to decrease the risk for NL in both cohorts $(P<0.0001)$, whereas other cardiovascular (CVD) drugs had no effect.

Conclusions: The risk for NL was increased by $50 \%$ in gout patients, compared to controls. Overall pattern of predictors was similar in gout patients and population controls. In patients with gout, male sex, diabetes mellitus (DM), obesity predicted $\mathrm{NL}$, whereas use of loop diuretics was protective. Overall, the most commonly used CVD drugs did not increase the risk for NL in patients with gout.

Disclosure of Interest: None declared

DOI: 10.1136/annrheumdis-2017-eular.2836

\section{THU0415 A STRUCTURED MONITORING PROGRAM FOR DRUG ALLERGY IN PATIENTS NEWLY INITIATED ON ALLOPURINOL}

B.Y. Thong ${ }^{1}$, K.-Y. Gan ${ }^{2}$, B.-H. Ang ${ }^{2} .{ }^{1}$ Rheumatology, Allergy and Immunology; ${ }^{2}$ Pharmacy, Tan Tock Seng Hospital, Singapore, Singapore

Background: Allopurinol allergy (drug eruption, severe cutaneous adverse reactions [SCAR] and drug induced hypersensitivity syndrome [DiHS]) develops during the first 2-12 weeks after initiation. SCAR risk factors include Chinese ethnicity, HLA-B*5801 positivity and chronic kidney disease. HLA-B*5801 testing to prevent SCAR has not been shown to be cost-effective in Singapore.

Objectives: To retrospectively study whether a structured monitoring program (SMP) can lead to early diagnosis of allopurinol allergy and prevent development of SCAR/DiHS.

Methods: SMP patients (cases) managed by rheumatologists were compared with controls managed by non-rheumatologists during the study period 1 Jan 2015 to 30 Jun 2016. Cases upon initiation of allopurinol had baseline full blood count (FBC), serum creatinine ( $\mathrm{Cr}$ ), alanine aminotransferase (ALT) and aspartate aminotransferase (AST) measured. If drug eruptions/abnormal laboratory tests developed during monitoring, allopurinol was stopped. The electronic dispensing system and computerized medical records were used for collection of patient demographics, indication for allopurinol use, initiation dose, monitoring intervals, laboratory results and clinical features of drug allergy. This was compared with the control group without an SMP. Chi square tests were used to compare differences in proportions and Mann-Whitney $U$ test for differences in medians. $P$ value $\leq 0.05$ was considered statistically significant.

Results: There were 61 cases and 30 controls with comparable age $(p=0.81)$, ethnicity ( $>80 \%$ Chinese) $(p=0.63)$ and estimated glomerular filtration rate, eGFR $(p=0.72)$. There were significantly more cases with tophaceous gout $(41 \%$ vs $10 \%, p=0.003)$, while more controls tumour lysis syndrome prophylaxis $(30 \%$ vs $0 \%, p<0.001$ ). Median (interquartile range, IQR) starting dose of 50 (50) mg was lower among cases versus controls of $100(200) \mathrm{mg}(\mathrm{p}<0.001)$; all cases had baseline and follow-up laboratory tests compared to controls $(p<0.001)$. Cases were followed up at a median (IQR) of 2 (1.1) weeks after initiation then 5 (2.0) weeks after the first visit, whereas controls were reviewed 8 (8.9) weeks after initiation, then 11 (4.6) weeks after the first visit. Two patients in the SMP group with normal eGFR developed maculopapular eruption (MPE), 1 elevated ALT/AST, and 1 both MPE and elevated ALT/AST within the first 14 days of initiation. One control with lymphoma and baseline eGFR $31 \mathrm{ml} / \mathrm{min} / 1.73 \mathrm{~m}^{2}$ developed DiHS (fever, MPE, elevated ALT/AST less than twice upper limit of normal) 43 days after initiation for tumour lysis prophylaxis. This occurred while on 2-weekly monitoring of FBC, ALT, AST. There were no cases of SCAR in both groups. 